\title{
SPECTRA OF SOME DOMAINS IN COMPACT LIE GROUPS AND THEIR APPLICATIONS ${ }^{1}$
}

\author{
HAJIME URAKAWA
}

\begin{abstract}
In this paper, we determine explicitly the spectra of the Dirichlet problems of some domains in simply connected compact simple Lie groups. As their applications, combining results of Hoffman [6] and Mori [10], we can state some stability conditions of these domains for the standard minimal isometric immersions into unit spheres.
\end{abstract}

1. Introduction and results. Let $M$ be a simply connected compact simple Lie group and let $T$ be its maximal torus. We give a bi-invariant Riemannian metric $g$ on $M$ from the Killing form $B$ of the Lie algebra $m$ of $M$ by

$$
g_{m}\left(X_{m}, Y_{m}\right)=-B(X, Y), \quad X, Y \in \mathfrak{m}, m \in M,
$$

where $X_{m}, Y_{m}$ are tangent vectors of $M$ at $m$ corresponding to $X, Y$. Let $d(x, y)$ be the distance of $(M, g)$ between two points $x, y$ in $M$. Then it is known (cf. Crittenden [4] and Sakai [13]) that the cut locus $C$ of the identity $e$ in $M$ satisfies

$$
C=\bigcup_{x \in M} x C(T) x^{-1},
$$

where $C(T)$ is the cut locus of $e$ in the flat torus $T$ induced from the Riemannian metric $g$. For a positive number $\varepsilon$ with $0<\varepsilon<d(e, C)$, consider a domain $\Omega(\varepsilon)$ containing the cut locus $C$ in $M$ defined by

$$
\Omega(\varepsilon)=\bigcup_{x \in M} x \Omega(\varepsilon, T) x^{-1}, \quad \Omega(\varepsilon, T)=\{t \in T ; d(t, C(T))<\varepsilon\} .
$$

These domains $\Omega(\varepsilon)$, which are invariant under all the inner automorphisms of $M$, shrink to the cut locus $C$ as $\varepsilon \rightarrow 0$.

Now let $\Delta$ be the Laplace-Beltrami operator of $(M, g)$ acting on the space $C^{\infty}(M)$ of smooth functions on $M$, and for every $\varepsilon$ with $0<\varepsilon<d(e, C)$, let us consider the following Dirichlet problem for the above domains:

$$
\begin{cases}\Delta u+\lambda u=0 & \text { on } M \backslash \overline{\Omega(\varepsilon)}, \\ u=0 & \text { on } \Omega(\varepsilon) .\end{cases}
$$

For a solution $u$ of the Dirichlet problem $(\#)_{\varepsilon}$, define a function $u^{0}$ on $M$ by

$$
u^{0}(x)=\int_{M} u\left(y x y^{-1}\right) d y,
$$

Received by the editors April 19, 1984 and, in revised form, March 4, 1985.

1980 Mathematics Subject Classification. Primary 58G25: Secondary 53C35.

Key words and phrases. Dirichlet problem, compact Lie groups, Laplace-Beltrami operators, zonal spherical functions.

${ }^{1}$ This work is supported by Max-Planck-Institut für Mathematik. 
where $d y$ is the Haar measure on $M$ normalized by $\int_{M} d y=1$. Then, if $u^{0}$ does not vanish identically, $u^{0}$ is also a solution of $(\#)_{\varepsilon}$ which is a zonal spherical function of $M$, i.e., invariant under all the inner automorphisms of $M$.

In this paper, we determine the spectra of the Dirichlet problem $(\#)_{\varepsilon}$, which have zonal spherical eigenfunctions as follows

THEOREM 1. Let $M$ be a simply connected compact simple Lie group and let $\Delta$ be the Laplace-Beltrami operator of the Riemannian metric $g$ of $M$ induced from the negative of the Killing form $B$ of the Lie algebra $m$ of $M$. Then for every $\varepsilon$ with $0<\varepsilon<d(e, C)$, the eigenvalues of the Dirichlet problem $(\#)_{\varepsilon}$ which have zonal spherical eigenfunctions are given by

$$
\left\{\frac{d(e, C)}{d(e, C)-\varepsilon}\right\}^{2}|\Lambda+\delta|^{2}-|\delta|^{2}, \quad \Lambda \in \mathbf{D},
$$

and the corresponding zonal spherical eigenfunctions $u_{\Lambda, \varepsilon}$ are described explicitly by

$$
u_{\Lambda, \varepsilon}(\exp H)= \begin{cases}\xi_{\Lambda+\delta}\left(\exp \left(\frac{d(e, C)}{d(e, C)-\varepsilon} H\right)\right) / \xi_{\delta}(\exp H), & \exp H \in T \backslash \Omega(\varepsilon, T), \\ 0, & \exp H \in \Omega(\varepsilon, T) .\end{cases}
$$

Here $\mathbf{D}$ is the set of all dominant integral forms on the Lie algebra $\mathrm{t}$ of $T, \delta$ is half the sum of all positive roots, $|\cdot|$ is the inner product of the dual space $\mathrm{t}^{*}$ of $\mathrm{t}$ induced from the negative of the Killing form, and $\xi_{\lambda}, \lambda \in \mathbf{D}$, are the alternating characters of $T$ (cf. §2).

Theorem 1 implies immediately

COROLlaRy 1. Under the assumptions of Theorem 1 , the first eigenvalue $\lambda_{1}(\varepsilon)$ of the Dirichlet problem $(\#)_{\varepsilon}, 0<\varepsilon<d(e, C)$, is given by

$$
\left\{\frac{d(e, C)}{d(e, C)-\varepsilon}\right\}^{2}|\delta|^{2}-|\delta|^{2}=\left\{\frac{d(e, C)}{d(e, C)-\varepsilon}\right\}^{2} \frac{d}{24}-\frac{d}{24},
$$

where $d=\operatorname{dim} M(c f .[15, p .291])$. The corresponding eigenfunction with the eigenvalue $\lambda_{1}(\varepsilon)$ is $u_{0, \varepsilon}$.

REMARK. In the case $S^{3}=\mathrm{SU}(2)$, the same formula as Theorem 1 was obtained in [3, p. 201]. Chavel and Feldman [3] also investigated the behavior of the eigenvalues $\lambda_{i}(\varepsilon)$ of the Dirichlet problems of the domains $X \backslash \overline{\Omega(\varepsilon)}$, where $\Omega(\varepsilon)=\{x \in$ $X ; d(x, Y)<\varepsilon\}$ for every compact Riemannian manifold $X$ and a closed submanifold $Y$ of $X$ with codim $\geqslant 2$. A more precise behavior of the first eigenvalue was obtained in Ozawa [12] and Matsuzawa and Tanno [9].

As a geometric application of Corollary 1, we can state some stability conditions of those domains $M \backslash \overline{\Omega(\varepsilon)}$ in $M$ for the standard minimal isometric immersions $x_{k}$ of $M$ into the unit sphere as follows.

Let $\left\{0=\lambda_{0}<\lambda_{1}<\lambda_{2}<\cdots<\lambda_{k}<\cdots\right\}$ be the set of all mutually distinct eigenvalues of the negative of the Laplace-Beltrami operator $\Delta$ acting on $C^{\infty}(M)$. 
Let $V^{k}, k=1,2, \ldots$, be the eigenspace with the eigenvalue $\lambda_{k}$, and put $m(k)+1$ $=\operatorname{dim} V^{k}$. We choose an orthonormal basis $\left\{f_{j}\right\}_{j=0}^{m(k)}$ of $V^{k}$ consisting of real valued functions with respect to the inner product $(\varphi, \psi)=\int_{M} \varphi(x) \psi(x) d \mu(x)$, where $d \mu(x)$ is the Haar measure of $M$ normalized by $\int_{M} d \mu(x)=m(k)+1$. Consider the mapping $x_{k}$ of $M$ into the Euclidean space $\mathbf{R}^{m(k)+1}$ defined by

$$
x_{k}(p)=\left(f_{0}(p), f_{1}(p), \ldots, f_{m(k)}(p)\right), \quad p \in M
$$

Then it turns out that the image of $x_{k}$ is contained in the unit sphere $S^{m(k)}$, moreover the mapping $x_{k}$ is a minimal isometric immersion of $\left(M, \lambda_{k} g / d\right)$, $d=\operatorname{dim} M$, into the unit sphere $S^{m(k)}$ with the standard Riemannian metric of constant curvature 1 (cf. [8]) since $M$ is a simple Lie group.

For a piecewise smooth domain $D$ in $M$, we call $D$ stable for the minimal immersion $x_{k}$ if, for all normal variations $D_{t}$ which fix the boundary $\partial D$, the function $V(t)=$ Volume $D_{t}$ satisfies $V^{\prime \prime}(0)>0$. Combining Corollary 1 with results of Hoffman [6] and Mori [10] we have

COROLlaRY 2. Under the situations of Theorem 1, if a positive number $\varepsilon$ satisfies

$$
d(e, C)>\varepsilon>d(e, C)-d(e, C)\left\{\frac{24 \lambda_{k}}{d}\left(\|A\|^{2}+d\right)+1\right\}^{-1 / 2},
$$

then $D$ is stable for the minimal isometric immersion $x_{k}$ for every $D \subset M \backslash \overline{\Omega(\varepsilon)}$. Here $\|A\|^{2}$ is the square of the length of the second fundamental form of the immersion $x_{k}$.

REMARK. In the case of $M=\operatorname{Sp}(n)$ and $k=1$, it is then known (cf. Nagura [11], Kobayashi and Takeuchi [7]) that $d=n(2 n+1)$,

$$
\|A\|^{2}=n(n-1)(n+1)(2 n+1), \quad \text { and } \quad \lambda_{1}=(2 n+1) /(4 n+4) .
$$

Therefore for every $D \subset M \backslash \overline{\Omega(\varepsilon)}, D$ is stable for the immersion $x_{1}$ if $d(e, C)>\varepsilon$ $>d(e, C)\{1-\sqrt{(n+1) /(7 n+1)}\}$, in particular, if $d(e, C)>\varepsilon>0.623 d(e, C)$.

2. Preliminaries. Since we will use the precise formula of the radial part (cf. [2]) of the Laplace-Beltrami operator and the structure of the cut loci $C$ and $C(T)$ (cf. [13]) in the proof of Theorem 1, we have to prepare some notation.

2.1. Let $M$ be a simply connected compact simple Lie group, and let $T$ be a maximal torus in $M$. Let $m$ (resp. $\mathfrak{t}$ ) be the Lie algebra of $M$ (resp. $T$ ). Since the Killing form $B$ is negative definite on $\mathrm{m}$, we define an $\operatorname{Ad}(M)$-invariant positive definite inner product $($,$) on \mathrm{m}$ by $(X, Y)=-B(X, Y), X, Y \in \mathrm{m}$, which induces a bi-invariant Riemannian metric $g$ on $M$ as in the introduction. Let $\Sigma$ be the root system of the complexification $\mathrm{m}^{\mathbf{C}}$ of $\mathrm{m}$ with respect to $\mathrm{t}$, i.e., the set of nonzero elements $\alpha$ of the dual space $\mathrm{t}^{*}$ of $\mathrm{t}$ such that $\left\{E \in \mathrm{m}^{\mathrm{C}} ;[H, E]=\sqrt{-1} \alpha(H) E\right.$ for all $H \in \mathrm{t}\}$ is not zero. We give a lexicographic order $>$ on $\Sigma$ and let $\Sigma_{+}$be the set of all positive roots. Let $\alpha^{0}$ be the highest root of $\Sigma_{+}$with respect to the order $>$. Put $\overline{\mathrm{t}^{+}}=\left\{H \in \mathrm{t} ; \alpha(H) \geqslant 0\right.$ for all $\left.\alpha \in \Sigma_{+}\right\}$. Then the cut locus $C$ of the identity $e$ in $(M, g)$ is given (cf. Sakai [13]) by

$$
C=\bigcup_{x \in M} x C(T) x^{-1}
$$


Here $C(T)$ is the cut locus of the flat torus $T$ induced from the Riemannian metric $g$ which is given (cf. Takeuchi [14] and Sakai [13]) by

$$
\begin{gathered}
C(T)=\exp \tilde{C}(\mathrm{t}), \\
\tilde{C}(\mathrm{t})=\bigcup_{s \in W} s\left\{H \in \overline{\mathrm{t}^{+}} ; \alpha^{0}(H)=2 \pi\right\},
\end{gathered}
$$

where $W$ is the Weyl group of $M$.

Put

$$
\tilde{D}^{+}(\mathrm{t})=\left\{H \in \overline{\mathrm{t}^{+}} ; \alpha^{0}(H) \leqslant 2 \pi\right\}, \quad \tilde{D}(\mathrm{t})=\bigcup_{s \in W} s \tilde{D}^{+}(\mathrm{t}),
$$

and $\tilde{D}=\bigcup_{x \in M} \operatorname{Ad}(x) \tilde{D}(t)$. Then $\tilde{C}(\mathrm{t})$ is the boundary $\partial \tilde{D}(\mathrm{t})$ of $\tilde{D}(\mathrm{t})$, both the exponential mappings exp: $\tilde{D}(\mathrm{t}) \rightarrow T$ and exp: $\tilde{D} \rightarrow M$ are onto mappings, and the restriction to the interior of $\tilde{D}$ is a diffeomorphism. Moreover the distance $d(e, C)$ between the identity $e$ and the cut locus $C$ is given by

$$
d(e, C)=2 \pi /\left|\alpha^{0}\right| \text {. }
$$

Here $|\cdot|$ is the norm of the inner product $($,$) on t^{*}$ induced from the inner product $($,$) on \mathrm{t}$ by $(\lambda, \mu)=\left(H_{\lambda}, H_{\mu}\right), \lambda, \mu \in \mathrm{t}^{*}$, where $H_{\lambda} \in \mathrm{t}, \lambda \in \mathrm{t}^{*}$, is the unique element in $\mathrm{t}$ satisfying $\left(H_{\lambda}, H\right)=\lambda(H)$ for every $H \in \mathrm{t}$. Note that the distance $d(x, y), x, y \in T$, coincides with the one with respect to the Riemannian metric on $T$ induced from the metric $g$ on $M$ (see Remark in [5, p. 80]). In fact, since $T$ is totally geodesic in $M$, we have only to show the existence of a distance minimizing geodesic in $T$ joining $e$ and every $x$ in $T$, but it follows immediately from Theorem 7.9(ii) and Lemma 7.10 in [5].

Then we have

LEMMA 2.1. For every $\varepsilon$ with $0<\varepsilon<d(e, C)=2 \pi /\left|\alpha^{0}\right|$,

(i) the set $\Omega(\varepsilon, T)=\{t \in T ; d(t, C(T))<\varepsilon\}$ is given by

$$
\begin{gathered}
\Omega(\varepsilon, T)=\exp \tilde{\Omega}(\varepsilon, \mathrm{t}), \\
\Omega(\varepsilon, \mathrm{t})=\bigcup_{s \in W} s\left\{H \in \overline{\mathrm{t}^{+}} ; 2 \pi\left(1-\varepsilon \frac{\left|\alpha^{0}\right|}{2 \pi}\right)<\alpha^{0}(H) \leqslant 2 \pi\right\} .
\end{gathered}
$$

(ii) The set $M \backslash \overline{\Omega(\varepsilon)}$ is given by

$$
M \backslash \overline{\Omega(\varepsilon)}=\bigcup_{x \in M} x \exp \tilde{D}^{+}(\varepsilon) x^{-1},
$$

where $\tilde{D}^{+}(\varepsilon)=\left\{H \in \overline{\mathrm{t}^{+}} ; \alpha^{0}(H)<2 \pi\left(1-\varepsilon\left|\alpha^{0}\right| / 2 \pi\right)\right\}$.

(iii) $d(e, C) /(d(e, C)-\varepsilon) \cdot \tilde{D}^{+}(\varepsilon)=\left\{H \in \overline{\mathrm{t}^{+}} ; \alpha^{0}(H)<2 \pi\right\}$. Here, for every $r>0, r \cdot \tilde{D}^{+}(\varepsilon)$ means the set $\left\{r H ; H \in \tilde{D}^{+}(\varepsilon)\right\}$.

Proof. (i) By the definition of $\tilde{\Omega}(\varepsilon, \mathfrak{t}),(2.3)$ and the invariance of the distance $d$ under the inner automorphisms of $M$, we have

$$
\tilde{\Omega}(\varepsilon, \mathrm{t})=\bigcup_{s \in W} s\left\{H \in \tilde{D}^{+}(\mathrm{t}) ; d(\exp H, \exp \tilde{C}(t))<\varepsilon\right\} .
$$

We denote $d_{e}(X, Y)=|X-Y|$ for $X, Y \in \mathrm{t}$. Then, for each $H \in \tilde{D}^{+}(\mathrm{t})$,

$$
\begin{aligned}
d(\exp H, \exp \tilde{C}(\mathrm{t})) & =d\left(\exp H, \exp \left(\tilde{C}(\mathrm{t}) \cap \overline{\mathrm{t}^{+}}\right)\right) \\
& =d_{e}\left(H, \tilde{C}(\mathrm{t}) \cap \overline{\mathrm{t}^{+}}\right) .
\end{aligned}
$$


In fact, putting $\Gamma=\{X \in \mathrm{t} ; \exp X=e\}$, we have

$$
d(\exp H, \exp \tilde{C}(\mathrm{t}))=d_{e}(H, \tilde{C}(\mathrm{t})+\Gamma)
$$

and

$$
d\left(\exp H, \exp \left(\tilde{C}(\mathrm{t}) \cap \overline{\mathrm{t}^{+}}\right)\right)=d_{e}\left(H,\left(\tilde{C}(\mathrm{t}) \cap \overline{\mathrm{t}^{+}}\right)+\Gamma\right) .
$$

Since $(\tilde{C}(\mathrm{t})+\Gamma) \cap \tilde{D}(\mathrm{t})=\tilde{C}(\mathrm{t})$ and $\left(\left(\tilde{C}(\mathrm{t}) \cap \overline{\mathrm{t}^{+}}\right)+\Gamma\right) \cap \tilde{D}(\mathrm{t})=\tilde{C}(\mathrm{t}) \cap \overline{\mathrm{t}^{+}}$,

$$
d_{e}(H, \tilde{C}(\mathrm{t})+\Gamma)=d_{e}(H, \tilde{C}(\mathrm{t}))
$$

and

$$
d_{e}\left(H,\left(C(\mathrm{t}) \cap \overline{\mathrm{t}^{+}}\right)+\Gamma\right)=d_{e}\left(H, \tilde{C}(\mathrm{t}) \cap \overline{\mathrm{t}^{+}}\right) .
$$

Since $H \in \tilde{D}^{+}(\mathrm{t})$, we have $d_{e}(H, \tilde{C}(\mathrm{t}))=d_{e}\left(H, \tilde{C}(\mathrm{t}) \cap \overline{\mathrm{t}^{+}}\right)$. For the proof of the second equality, choose an element $X$ in $\tilde{C}(\mathrm{t}) \cap \overline{\mathrm{t}^{+}}$such that

$$
d\left(\exp H, \exp \left(\tilde{C}(\mathrm{t}) \cap \overline{\mathrm{t}^{+}}\right)\right)=d(\exp H, \exp X) .
$$

Then $d(\exp H, \exp X)=d(e, \exp (-H+X))$ and $-H+X \in \tilde{D}(\mathrm{t})$, because $0 \leqslant$ $\alpha(H), \alpha(X) \leqslant 2 \pi$ for $\alpha \in \Sigma_{+}$, and the definition (2.4) of $\tilde{D}(t)$. Then

$$
d(e, \exp (-H+X))=|-H+X|
$$

which implies $d\left(\exp H, \exp \left(\tilde{C}(\mathrm{t}) \cap \overline{\mathfrak{t}^{+}}\right)\right) \geqslant d_{e}\left(H, \tilde{C}(\mathrm{t}) \cap \overline{\mathfrak{t}^{+}}\right)$. The converse inequality is clear.

By (2.3) and (2.6), we have

$$
\begin{aligned}
\left\{H \in \tilde{D}^{+}(\mathrm{t})\right. & ; d(\exp H, \exp \tilde{C}(\mathrm{t}))<\varepsilon\}=\left\{H \in \tilde{D}^{+}(\mathrm{t}) ; d_{e}\left(H, \tilde{C}(\mathrm{t}) \cap \overline{\mathrm{t}^{+}}\right)<\varepsilon\right\} \\
= & \left\{(1-r) \frac{2 \pi H_{\alpha^{0}}}{\left(\alpha^{0}, \alpha^{0}\right)}+X ;|r|<\frac{\varepsilon\left|\alpha^{0}\right|}{2 \pi}, X \in \mathrm{t}, \alpha^{0}(X)=0\right\} \cap \tilde{D}^{+}(t) \\
= & \left\{H \in \overline{\mathrm{t}^{+}} ; 2 \pi\left(1-\varepsilon \frac{\left|\alpha^{0}\right|}{2 \pi}\right)<\alpha^{0}(H) \leqslant 2 \pi\right\} .
\end{aligned}
$$

For (ii), we have only to show $X=Y$ when $g_{1} \exp X g_{1}^{-1}=g_{2} \exp Y g_{2}^{-1}, X \in \tilde{D}^{+}(\varepsilon)$, $Y \in \tilde{D}^{+}(\mathrm{t}), g_{1}, g_{2} \in M$. But in this case, we have $\exp X=\exp s Y$ for some $s \in W$ by Lemma 7.10 in [5]. Since $s Y \in \tilde{D}(\mathrm{t})$ and $X \in \tilde{D}^{+}(\varepsilon)$, $\exp X=\exp s Y$ implies $X=s Y$, and then $X=Y$. (iii) follows immediately from (ii). Q.E.D.

2.2. For $\lambda \in \mathrm{t}^{*}, \lambda \neq 0$, put $H_{\lambda}^{*}=2 H_{\lambda}(\lambda, \lambda)$. Then since $M$ is simply connected, the lattice $\Gamma=\{H \in \mathrm{t} ; \exp H=e\}$ is given by $\Gamma=2 \pi \sum_{i=1}^{\prime} \mathbf{Z} H_{\alpha_{i}}^{*}$, where $\left\{\alpha_{i}\right\}_{i=1}^{l}$ is a fundamental system of $\Sigma$ with respect to the order $>$, and $l=\operatorname{dim} T$. Put

$$
\begin{aligned}
I & =\left\{\lambda \in \mathrm{t}^{*} ; \lambda\left(H_{\alpha_{i}}^{*}\right) \in \mathbf{Z}, i=1, \ldots, l\right\} \\
& =\left\{\lambda \in \mathrm{t}^{*} ; \lambda(\Gamma) \subset 2 \pi \mathbf{Z}\right\}, \\
\mathbf{D} & =\left\{\lambda \in I ;(\lambda, \boldsymbol{\alpha}) \geqslant 0 \text { for every } \alpha \in \Sigma^{+}\right\} .
\end{aligned}
$$

An element of $\mathbf{D}$ is called a dominant integral form on $\mathrm{t}$. For $\lambda \in I$, define a function $\xi_{\lambda}$ on $T$, called the alternating character, by

$$
\xi_{\lambda}(\exp H)=\sum_{s \in W}(-1)^{s} e^{s \lambda(H)}, \quad H \in \mathrm{t} .
$$


Put $\delta=\frac{1}{2} \sum_{\alpha \in \Sigma^{+}} \alpha$. Then $\delta$ belongs to $\mathbf{D}$. Moreover it is known that

$$
\xi_{\delta}(\exp H)=\prod_{\alpha \in \Sigma^{+}}\left(e^{\sqrt{-1} \alpha(H) / 2}-e^{-\sqrt{-1} \alpha(H) / 2}\right),
$$

every $\xi_{\lambda}, \lambda \in I$, can be divided by $\xi_{\delta}$, and $\xi_{\Lambda+\delta} / \xi_{\delta}, \lambda \in \mathbf{D}$, coincides with the restriction to $T$ of the character $\chi_{\Lambda}$ of the irreducible unitary representation of $M$ with highest weight $\Lambda$ (cf. [14]). For every $C^{\infty}$ zonal spherical function $f$ on $M$, let $\bar{f}$ be its restriction to $T$. Then $\bar{f}(\exp s H)=\bar{f}(\exp H), s \in W, H \in \mathrm{t}$, and we have (cf. Berezin [2] or [14])

$$
\xi_{\delta}(\overline{\Delta f})=\left\{\Delta_{0}+|\delta|^{2}\right\}\left(\xi_{\delta} \bar{f}\right)
$$

on $T$, where $\Delta_{0}$ is the standard Laplacian on $T$ induced from the Euclidean Laplacian of $\mathrm{t}$ with respect to the inner product (, ).

3. Proof of Theorem 1. For $0<\varepsilon<d(e, C)$, assume that a zonal spherical function $u$ on $M$ satisfies

$$
\begin{cases}\Delta u+\lambda u=0 & \text { on } M \backslash \overline{\Omega(\varepsilon)} \\ u=0 & \text { on } \Omega(\varepsilon)\end{cases}
$$

Then by (2.7) we have

$$
\begin{cases}\left(\Delta_{0}+|\delta|^{2}\right)\left(\xi_{\delta} \bar{u}\right)+\lambda \xi_{\delta} \bar{u}=0 & \text { on } T \backslash \overline{\Omega(\varepsilon, T)} \\ \bar{u}=0 & \text { on } \Omega(\varepsilon, T) .\end{cases}
$$

Now define a function $\left(\xi_{\delta} \bar{u}\right)_{\varepsilon}$ on $T$ by

$$
\left(\xi_{\delta} \bar{u}\right)_{\varepsilon}(\exp H)=\left(\xi_{\delta} \bar{u}\right)\left(\exp \left(\frac{d(e, C)-\varepsilon}{d(e, C)} H\right)\right), \quad H \in \tilde{D}(\mathrm{t}) .
$$

It is well defined on $T$ because of Lemma 3.1(iii), and $\bar{u}=0$ on $\Omega(\varepsilon, T)$. Also define a function $\overline{\left(\xi_{\delta} \bar{u}\right)_{\varepsilon}}$ on $\tilde{D}(\mathrm{t})$ by

$$
\widetilde{\left(\xi_{\delta} \bar{u}\right)_{\varepsilon}}(H)=\left(\xi_{\delta} \bar{u}\right)_{\varepsilon}(\exp H), \quad H \in \tilde{D}(\mathrm{t}) .
$$

Then the function $\overline{\left(\xi_{\delta} \bar{u}\right)_{\varepsilon}}$ satisfies

$$
\left\{\begin{array}{l}
\Delta_{0} \overline{\left(\xi_{\delta} \bar{u}\right)_{\varepsilon}}+\left\{\frac{d(e, C)-\varepsilon}{d(e, C)}\right\}^{2}\left(|\delta|^{2}+\lambda\right) \overline{\left(\xi_{\delta} \bar{u}\right)_{\varepsilon}}=0, \\
\text { on the interior of } \tilde{D}(t),
\end{array}\right.
$$

Moreover, $\overline{\left(\xi_{\delta} \bar{u}\right)_{\varepsilon}}=0$ on $\tilde{D}^{+}(\mathrm{t})$ since $\xi_{\delta}=0$ on $\partial \tilde{D}^{+}(\mathrm{t})$. Therefore $\overline{\left(\xi_{\delta} \bar{u}\right)_{\varepsilon}}$ is the eigenfunction of the Dirichlet problem for the domain $\tilde{D}^{+}(t)$. Since the domain $\tilde{D}^{+}(\mathrm{t})$ is a fundamental domain of the affine Weyl group of the Lie group $M$ acting on $t$, by a theorem of Bérard [1], we have

$$
\overline{\left(\xi_{\delta} \bar{u}\right)_{\varepsilon}}(H)=\sum_{s \in W}(-1)^{s} e^{\sqrt{-1} s(\Lambda+\delta)(H)}
$$


for some $\Lambda \in \mathbf{D}$, and $\{(d(e, C)-\varepsilon) / d(e, C)\}^{2}\left(|\delta|^{2}+\lambda\right)=|\Lambda+\delta|^{2}$. Therefore we obtain

$$
\lambda=\left\{\frac{d(e, C)}{d(e, C)-\varepsilon}\right\}^{2}|\Lambda+\delta|^{2}-|\delta|^{2}
$$

and

$$
u(\exp H)=\left\{\begin{array}{l}
\xi_{\Lambda+\delta}\left(\exp \left(\frac{d(e, C)}{d(e, C)-\varepsilon} H\right)\right) / \xi_{\delta}(\exp H), \quad H \in \Omega(\varepsilon, \mathrm{t}), \\
0, \quad H \notin \Omega(\varepsilon, \mathrm{t}) .
\end{array}\right.
$$

Conversely, the function $u$ defined by (3.3) is a zonal spherical function on $M$ and satisfies (3.1) with the eigenvalue (3.2). We have proved Theorem 1.

\section{REFERENCES}

1. P. H. Bérard, Spectres et groupes cristallographiques I: Domaines euclidiens, Invent. Math. 58 (1980), 179-199.

2. F. A. Berezin, Laplace operators on semi-simple Lie groups, Trudy Moskov. Mat. Obshch. 6 (1957), 371-463.

3. I. Chavel and E. A. Feldman, Spectra of domains in compact manifolds, J. Funct. Anal. 30 (1978), 198-222.

4. R. Crittenden, Minimum and conjugate points in symmetric spaces, Canad. J. Math. 14 (1962), 320-328.

5. S. Helgason, Differentiai geometry, Lie groups and symmetric spaces, Academic Press, New York, 1978.

6. D. Hoffman, Lower bounds on the first eigenvalue of the Laplacian of Riemannian submanifolds, Minimal Submanifolds and Geodesic, Kaigai Publ., Tokyo, 1978, pp. 61-73.

7. S. Kobayashi and M. Takeuchi, Minimal imbeddings of R-spaces, J. Differential Geom. 2 (1968), 203-215.

8. K. Mashimo, Degree of the standard isometric minimal immersion of complex projective spaces into spheres, Tsukuba J. Math. 4 (1980), 133-145.

9. T. Matsuzawa and S. Tanno, Estimates of the first eigenvalue of a big cup domain of a 2-sphere, Compositio Math. 47 (1982), 95-100.

10. H. Mori, Notes on the stability of minimal submanifolds of Riemannian manifolds, Yokohama Math. J. 25 (1977), 9-15.

11. T. Nagura, On the lengths of the second fundamental forms of R-spaces, Osaka J. Math. 14 (1977), 207-223.

12. S. Ozawa, The first eigenvalue of the Laplacian on two dimensional Riemannian manifolds, Tôhoku Math. J. 34 (1982), 7-14.

13. T. Sakai, On the structure of cut loci in compact Riemannian symmetric spaces, Math. Ann. (2) 235 (1978), 129-148.

14. M. Takeuchi, Modern theory of spherical functions, Iwanami, Tokyo, 1975 (in Japanese).

15. H. Urakawa, The Heat equations on compact Lie groups, Osaka J. Math. 12 (1975), 285-297.

Department of Mathematics, College of General Education, TOHoku University, Kawauchi, SENDAI, 980, JAPAN

Max-Planck-Institut fur Mathematik, Gottfried-Claren-Strasse 26, 5300, Bonn 3, Federal REPUBLIC OF GERMANY 